\title{
Endocrine
}

\section{Is the hypothalamic-pituitary-adrenal axis disrupted in type 2 diabetes mellitus and is this relevant for bone health? --Manuscript Draft--}

\begin{tabular}{|c|c|}
\hline Manuscript Number: & ENDO-D-16-01061R1 \\
\hline Full Title: & $\begin{array}{l}\text { Is the hypothalamic-pituitary-adrenal axis disrupted in type } 2 \text { diabetes mellitus and is } \\
\text { this relevant for bone health? }\end{array}$ \\
\hline Article Type: & Letter to the Editor \\
\hline Corresponding Author: & $\begin{array}{l}\text { lacopo Chiodini } \\
\text { Fondazione IRCCS Ca' Granda-Ospedale Maggiore Policlinico } \\
\text { ITALY }\end{array}$ \\
\hline \multicolumn{2}{|l|}{$\begin{array}{l}\text { Corresponding Author Secondary } \\
\text { Information: }\end{array}$} \\
\hline Corresponding Author's Institution: & Fondazione IRCCS Ca' Granda-Ospedale Maggiore Policlinico \\
\hline \multicolumn{2}{|l|}{$\begin{array}{l}\text { Corresponding Author's Secondary } \\
\text { Institution: }\end{array}$} \\
\hline First Author: & Cristina Eller-Vanicher \\
\hline \multicolumn{2}{|l|}{ First Author Secondary Information: } \\
\hline \multirow[t]{3}{*}{ Order of Authors: } & Cristina Eller-Vanicher \\
\hline & Alfredo Scillitani \\
\hline & lacopo Chiodini \\
\hline \multicolumn{2}{|c|}{ Order of Authors Secondary Information: } \\
\hline \multicolumn{2}{|l|}{ Funding Information: } \\
\hline Abstract: & No abstract required \\
\hline \multirow[t]{2}{*}{ Response to Reviewers: } & $\begin{array}{l}\text { Reviewer \#1: The letter to the editor "Is the hypothalamic-pituitary-adrenal axis } \\
\text { disrupted in type } 2 \text { diabetes mellitus and is this relevant for bone health?" is well } \\
\text { written and makes an important contribution to the literature on the future directions } \\
\text { investigating the role of hypothalamic-pituitary-adrenal axis and type } 2 \text { diabetes with } \\
\text { bone metabolism and fracture risk. } \\
\text { We thank the reviewer for the appreciation. }\end{array}$ \\
\hline & $\begin{array}{l}\text { Reviewer \#2: General comment: } \\
\text { The authors commented about several papers published in Endocrine and further } \\
\text { discussed the relevance of the disruption of the HPA axis in DM2 patients for the } \\
\text { metabolic control and in addition to the bone function and health. They mention several } \\
\text { studies related to different factors that modulate glucocorticoid activity at bone tissue } \\
\text { including the new } 11 \text { HSD1 inhibitors as potential candidates to ameliorate glucose } \\
\text { metabolism and additionally to improve osteoblast biology. } \\
\text { This is an interesting point and brief discussion. } \\
\text { Specific comments: } \\
\text { Q: It is suggested to include a brief paragraph mentioning the action mechanisms by } \\
\text { which glucocorticoids might damage the bone, in order to get in context the whole } \\
\text { discussion. Please, pay some attention to dissect those direct effects of glucocorticoids } \\
\text { in the bone to those due to the diabetic metabolic alterations or its complications in } \\
\text { other organs (nephropathy, micro/macro-angiopathy, neuropathy,...). } \\
\text { A: We agree with the referee. Accordingly, a paragraph summarizing the } \\
\text { glucocorticoids effect on bone has been added (page 2, lines } 31-49 \text { ). In addition, the } \\
\text { presence of cortical porosity in T2D related osteoporosis has been discussed (page 2, } \\
\text { lines 53-58) }\end{array}$ \\
\hline
\end{tabular}


Q: Commenting a bit further in deep how the glucocorticoid receptor polymorphisms might affect glycemic control and bone function would be of interest.

A: We agree. A paragraph briefly summarizing the possible role of glucocorticoid receptor polymorphisms has been added (page 3, lines 7-22)

Q: Lines 40-44. "Thirdly, recent data showed that, as compared with subjects without T2D, even in T2D patients with good metabolic control the cortisol secretion is enhanced (although still normal as evaluated by the commonly used parameters of cortisol secretion)..." Did the authors mean that T2D patients have cortisol levels in the upper limit of the normal range? Or that common methodology is not adequate to measure cortisol levels in T2D patients? Please, clarify and write it accordingly. A: We meant that T2D patients have cortisol levels in the upper limit of the normal range. This is now stated on page 3 (line 2).

Q: Line 47. "...the degree of cortisol secretion...". "degree" might be suppressed. Several times repeated along the text.

A: We agree. The requested change has been made.

Q: Line 51. Correct "glycometbolic".

A: We apologize for the typos. The error has been corrected (page 3, line 9)

Q: Line 58. What "hormonal parameters" are the authors referring?

A: We meant that the basal cortisol homeostasis and androgens levels were not affected by the 11-ßhydroxysteroid dehydrogenase type 1 inhibitor. This is now clarified in the new version of the manuscript (page 3, line 29)

Reviewer \#3: Comments on letter to the editor (ENDO-D-1601061)

$Q$ : The letter to the editor written by Eller-Vainicher and colleagues is dealing with an intriguing topic, which is the HPA-axis alterations in type-2 diabetes and their potential implications for osteoporosis and fractures. The letter is clear and well-written. I have only a general comment. In the view of the authors, it seems that osteoporosis in diabetes may be a consequence of HPA-axis disruption. Despite some evidence provide support to the occurrence of alterations of indices of HPA-axis activity in poorly-controlled diabetes, it is still debatable whether or not those alterations may have clinical relevance. It should be considered that osteoporosis is a multifactorial disease and, because of this, the potential causative effects of alteration of cortisol secretion on osteoporosis and fracture risk is difficult to be understood. Considering that there are still no solid proofs regarding the etiology of hypercortisolism in type-2 diabetes, the suggestion of using 11 beta-HSD inhibitors as an additional therapy for osteoporosis is largely empirical. Even if disruption of cortisol secretion may have a role in determining osteoporosis in type-2 diabetes, we still do not know whether lowering cortisol secretion rate is more beneficial than a strict metabolic control of diabetes.

A: We completely agree and thank the reviewer for this comment. The point raised has been discussed in the new version of the manuscript (page 3, lines 40-54)

\section{ASSOCIATE EDITOR:}

Q: Please check Instructions for Authors related to "Letter to the Editor": Text is limited to 750 words, with no abstract. There may be 1 figure, up to 3 references, ...

A: The manuscript now satisfies the Instructions for Authors related to "Letter to the Editor" 
Letter to the Editor: Is the hypothalamic-pituitary-adrenal axis disrupted in type 2 diabetes mellitus and is this relevant for bone health?

Authors: Cristina Eller-Vainicher ${ }^{1}$, Alfredo Scillitani ${ }^{2}$, Iacopo Chiodini ${ }^{1}$.

Affiliations: ${ }^{1}$ Unit of Endocrinology and Metabolic Diseases, IRCCS Cà Granda-Ospedale Maggiore

Policlinico Milan, Italy. ${ }^{2}$ Unit of Endocrinology, “Casa Sollievo della Sofferenza”, Hospital, IRCCS, San Giovanni Rotondo, Foggia, Italy.

Key terms: osteoporosis, cortisol secretion, type 2 diabetes

Corresponding author and person to whom the reprint request should be addressed:

Iacopo Chiodini, MD

Unit of Endocrinology and Metabolic Diseases, Padiglione Granelli,

Fondazione IRCCS Cà-Granda, Ospedale Maggiore Policlinico

Via Francesco Sforza 35, 20122 Milan, Italy.

Phone : +39-2-55033355 ; Fax : +39-2-50320605; e-mail: iacopo.chiodini@unimi.it 
We read with interest the Editorial (1) and the two studies recently published on this Journal $(2,3)$ regarding the hypothalamic-pituitary-adrenal axis disruption in type 2 diabetes mellitus (T2D) and its consequences on the glycometabolic control.

We agree with Raff and Magill that it would be prudent to screen for a condition of subtle hypercortisolism all patients with poorly controlled T2D. Beside this consideration, we would like to focus on the fact that, in addition to the metabolic control, the degree of cortisol secretion in T2D (even within the normal range) might be associated with the T2D related bone damage. Interestingly, there are interrelations and similarities between T2D and the condition of hypercortisolism not only regarding the pathogenesis of the glucose metabolism alteration in both conditions, but possibly also regarding the pathogenesis of the bone tissue alterations.

Firstly, osteoporosis in T2D is characterized by a low bone turnover with a normal/decreased bone resorption and decreased bone apposition, partially due to the inhibition of the $\mathrm{Wnt} / \beta$-catenin signaling. Indeed, this pathway is a critical regulator of osteoblastogenesis and in the T2D related osteoporosis its inhibition may lead to a preferential differentiation of the mesenchymal cells toward adipocytes than toward osteoblasts. Similarly, in the glucocorticoid (GC) induced osteoporosis (GIO) the osteoblast differentiation is inhibited, due to a mesenchymal cells shift toward cells of the adipocytic lineage instead of toward osteoblasts. In addition, the GC excess decrease osteoblastic function and promote osteoblast and osteocyte apoptosis. This latter effect induces bone microarchitectural alterations, reducing bone quality. During the initial period of GC excess bone resorption is increased due to the enhanced levels of the receptor activator of nuclear factor kappa-B ligand and the decreased osteoprotegerin production in osteoblasts. As the GC exposure continues, bone remodeling decreases and the inhibition of bone apposition becomes the main pathogenetic mechanism of skeletal damage. Finally, the occurrence of an enhanced porosity of the cortical bone has been described as a further mechanism increasing the fracture risk in GIO.

Secondly, in T2D patients, the diabetic chronic complications and the scarce glycometabolic control seem to be associated with the hip fracture risk. Indeed, the cortical porosity and the fracture risk are particularly increased in patients with T2D chronic complications. Interestingly, these latter patients have also higher cortisol levels than patients with not complicated T2D. 
Thirdly, recent data show that, as compared with not diabetic subjects, in T2D patients (even in those with good metabolic control) the cortisol secretion is in the upper limit of the normal range, bone turnover is reduced and the vertebral fractures prevalence is associated with both cortisol secretion and the N363S sensitizing variant of the glucocorticoid receptor (GR) gene. Interestingly, the GR gene polymorphisms have been found to potentially influence both glycometabolic control and bone function. Indeed, the N363S polymorphism and the Bcl1 intronic mutation in the GR gene seem to increase GC sensitivity. These polymorphic variants (in heterozygosis for N363S and in homozygosis for Bcl1) seem to be associated with a worse metabolic profile and a decreased spinal and trochanteric bone density. However, an increased sensitivity of the GR should lower the circulating cortisol levels, so that their action at the GR should be correspondingly reduced. However, the GC sensitivity of bone may be increased in the conditions of endogenous or exogenous hypercortisolism.

Finally, an agent inhibiting the 11-ßhydroxysteroid dehydrogenase type 11 (11HSD1) activity, named INCB13739, has been found to ameliorate the glycometabolic control, without negatively affecting the basal cortisol homeostasis and androgens levels. Furthermore, animal and in vitro studies suggested the potential role of a 11HSD1 inhibitor in stimulating osteoblast differentiation.

These considerations may be of clinical interest. Indeed, if osteoporosis in T2D was related, at least partially, to the degree cortisol secretion and/or sensitivity, the treatment with an 11HSD1 inhibitor could improve diabetic control and reduce the fracture risk at the same time.

However, since the majority of the available studies are cross-sectional, it is still unclear if the alterations of the cortisol secretion may really influence the control of T2D. Furthermore, osteoporosis is a multifactorial disease and, therefore, the possible causative effects of the disrupted cortisol secretion on bone health is difficult to be understood. Finally, even if the altered cortisol secretion may contribute to the pathogenesis of the osteoporosis in T2D, it is to be determined whether lowering cortisol secretion rate is more beneficial for bone than a strict glycometabolic control. Therefore, the suggestion of using 11HSD inhibitors as a potential therapy for T2D related osteoporosis is, at the moment, entirely speculative. 
Funding: none

\section{Conflict of Interest:}

Cristina Eller-Vainicher declares that he has no conflict of interest.

Alfredo Scillitani declares that he has no conflict of interest.

Iacopo Chiodini declares that he has no conflict of interest.

Ethical approval: This article does not contain any studies with human participants performed by any of the authors. 


\section{References}

1. H. Raff, S.B. Magill. Is the hypothalamic-pituitary-adrenal axis disrupted in type 2 diabetes mellitus? Endocrine 54(1):273-275 (2016).

2. E.K. Spanakis, X. Wang, B.N. Sanchez, A.V. Diez Roux, B.L. Needham, G.S. Wand, T. Seeman, S.H. Golden, Lack of significant association between type 2 diabetes mellitus with longitudinal change in diurnal salivary cortisol: the multiethnic study of atherosclerosis. Endocrine 53(1), 227239 (2016).

3. G. Bellastella, M.I. Maiorino, A. De Bellis, M.T. Vietri, C.Mosca, L. Scappaticcio, D. Pasquali, K. Esposito, D. Giugliano. Serum but not salivary cortisol levels are influenced by daily glycemic oscillations in type 2 diabetes. Endocrine 53(1), 220-226 (2016). 